\section{DRUG COATED BALLOON ONLY ANGIOPLASTY FOR STENT THROMBOSIS}

${ }^{1}$ Ioannis Merinopoulos, ${ }^{2}$ Tharusha Gunawardena, ${ }^{2}$ Natasha Corballis, ${ }^{3}$ Upul Wickramarachchi, ${ }^{3}$ Clint Maart, ${ }^{3}$ Sulfi Sreekumar, ${ }^{3}$ Chris Sawh, ${ }^{3}$ Trevor Wistow, ${ }^{3}$ Toomas Sarev, ${ }^{3}$ Tim Gilbert, ${ }^{3}$ Alisdair Ryding, ${ }^{4}$ Vassilios Vassiliou, ${ }^{3}$ Simon Eccleshall. ${ }^{1}$ University of East Anglia, Norfolk \& Norwich University Hospital, Norwich, UK; ${ }^{2}$ Norwich Medical School and Norfolk \& Norwich University Hospital; ${ }^{3}$ Norfolk \& Norwich University Hospital; ${ }^{4}$ Norwich Medical School, University of East Anglia

\subsection{6/heartjnl-2021-BCS.57}

Introduction Stent thrombosis, with a 5-45\% mortality and $15-20 \%$ recurrence rate at 5 years, represents the most severe end of the stent failure spectrum. There are no data regarding the outcomes of patients with stent thrombosis (ST) being treated with paclitaxel drug coated balloon (DCB) angioplasty. Our aim was to determine the all-cause mortality of patients treated with DCB angioplasty for ST.

Methods We identified all patients treated for ST between June 2011 and November 2019. We excluded patients who died in the cath lab, patients with uncrossable lesions and patients who were treated with a staged CABG. The primary endpoint was all-cause mortality. The secondary endpoint was target lesion revascularisation (TLR). Survival data were obtained through the UK Health and Social Care Information Service. Clinical and angiographic data were collected from our prospectively collated database supplemented with data from electronic records where required. All angiograms were reviewed by two experienced operators to confirm ST, TIMI flow pre- and post-intervention and identify bifurcation lesions.

\section{Abstract 57 Table 1}

\begin{tabular}{|l|l|l|}
\hline & Hazard ratio $(95 \%$ CI $)$ & P value \\
\hline Cardiogenic shock & $5.28(2.029,13.744)$ & 0.001 \\
\hline AF & $4.89(1.753,13.643)$ & 0.002 \\
\hline eGFR & $0.98(0.959,0.994)$ & 0.008 \\
\hline
\end{tabular}

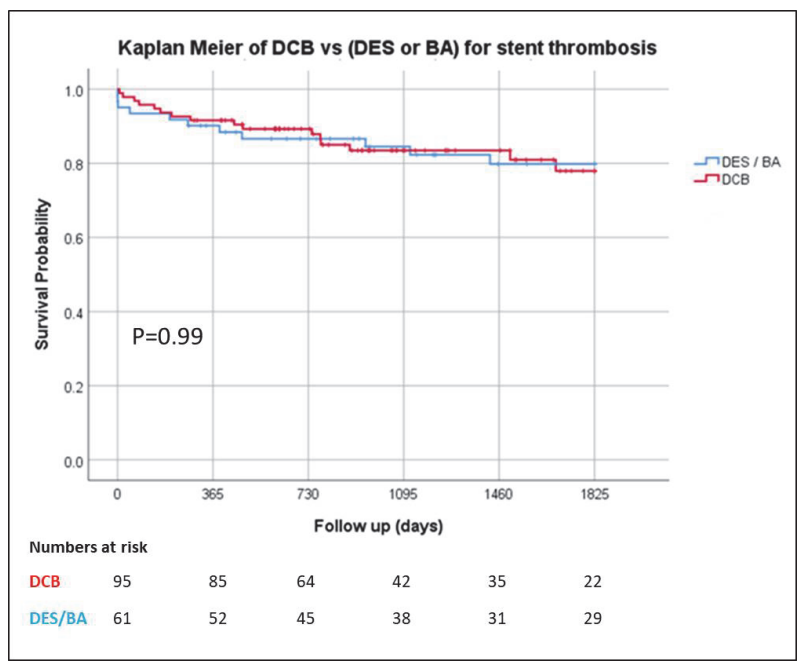

Abstract 57 Figure 1

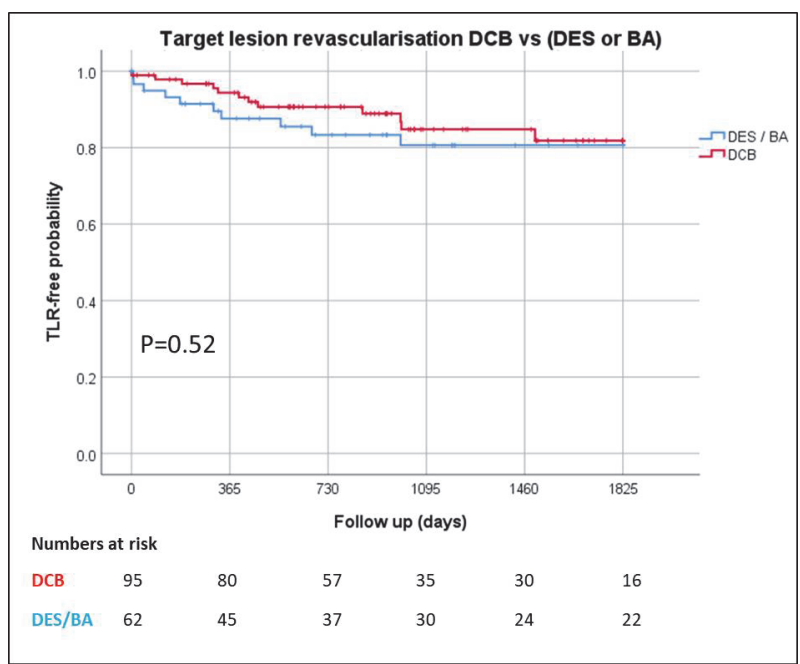

Abstract 57 Figure 2

Results A total of 178 patients were identified; 95 treated with DCB, 35 with balloon angioplasty (BA), 26 with drug eluting stent (DES), 19 with combination of DES and DCB and 3 with thromboaspiration only. The average age was $66.2 \pm 10.5$ years old, while male patients accounted for 76.8\%. There were a few differences between DCB, BA and DES. The DES group had more patients with history of CABG, hypertension and lower average eGFR at presentation while the DCB group had more patients with history of smoking. Importantly, the great majority of patients treated with DCB had very late ST. The patients were followed-up for an average of $45.6 \pm 29.8$ months; 39.8 $\pm 25.4,49.8$ $\pm 28.1,57.1 \pm 40.9$ months for the DCB, BA and DES groups respectively. All-cause mortality at 9 months was $8.5 \%$ for whole cohort; 7.4\%, 2.9\%, 19.2\% for the DCB, BA and DES groups respectively (DCB vs DES $p=0.07$; DCB vs BA $\mathrm{p}=0.34$; DES vs BA $\mathrm{p}=0.03$ ). Table 1 shows the only independent predictors of mortality up to 5 years in multivariable Cox regression analysis. When considering only the patients discharged alive, all-cause mortality at 9 months was $4.7 \%$ for whole cohort; 5.4\%, 2.9\%, 8.7\% for DCB, BA and DES groups respectively (no statistical difference between any pairs). There was no statistical difference in all-cause mortality (figure 1) or local TLR (figure 2) up to 5 years of follow-up.

Conclusion DCB-only angioplasty for patients with ST is safe and may be considered as a treatment option especially in patients with very late ST.

Conflict of Interest no conflict of interest

\section{PERCUTANEOUS CORONARY INTERVENTION IN PATIENTS TURNED DOWN FOR SURGICAL REVASCULARIZATION: A SINGLE-CENTRE EXPERIENCE}

Mohaned Egred, Abdalazeem Ibrahem, Ayman Al-atta, Ahmed Abdalwahab, Mohamed Farag. Freeman Hospital, Newcastle upon Tyne, UK

\subsection{6/heartjnl-2021-BCS.58}

Aim We aimed to evaluate the reasons for surgical ineligibility and in-hospital outcome of percutaneous coronary intervention (PCI) in these patients at a large tertiary centre. 
Methods We performed a retrospective analysis of prospectively collected data on surgical turndown patients who underwent PCI between April 2013 and November 2020. Data were collected from the institutional electronic database.

Results Of 473 patients, $69.8 \%$ were male with mean age of $72 \pm 11$ years and mean body mass index of $29 \pm 6 \mathrm{~kg} / \mathrm{m} 2$. Turndown reasons were documented in $52.4 \%$ of patients and mainly included the perceived frailty of the patient with associated comorbidities and/or the quality of distal coronary arteries. Elective cases with stable angina constituted 216 patients $(45.7 \%)$ and urgent cases with acute coronary syndromes constituted 257 patients (54.3\%). Mean hospital stay was $4 \pm 5$ days. Procedural success was documented in 457 out of 473 patients (96.6\%). Adjunctive tools included physiological assessments in 34 patients (7.2\%), intracoronary imaging in 97 patients (20.5\%), rotational atherectomy in 96 patients $(20.3 \%)$, laser atherectomy in 12 patients $(2.5 \%)$ and lithotripsy in 3 patients $(0.6 \%)$. In-hospital complications included major adverse cardiac events in 32 patients $(6.8 \%)$, death in 12 patients $(2.5 \%)$, myocardial infarction in 21 patients $(4.4 \%)$, ischaemic stroke in 1 patient $(0.2 \%)$, coronary perforation in 7 patients $(1.5 \%)$, repeat target vessel revascularization in 6 patients $(1.3 \%)$, major access-site bleeding in 2 patients $(0.4 \%)$, aortic dissection in 1 patient $(0.2 \%)$ and new acute kidney injury requiring dialysis in 1 patient $(0.2 \%)$.

Conclusions In real-world data, the process of determining suitability for surgical revascularization is often complex. PCI in surgically ineligible patients is generally safe and effective.

Conflict of Interest None

\section{PRASUGREL LOADING FOR PATIENTS WITH HIGH BLEEDING RISK PRESENTING WITH ST-SEGMENT ELEVATION MYOCARDIAL INFARCTION REFERRED FOR PRIMARY PERCUTANEOUS INTERVENTION - A RETROSPECTIVE ANALYSIS OF BLEEDING AND MORTALITY}

Theofanis Nizamis, Nikesh Jathanna, Charlotte Leeson, Sachin Jadhav. Nottingham University Hospitals NHS Trust, Nottingham, UK

\subsection{6/heartjnl-2021-BCS.59}

Background and introduction Prasugrel is an ideal antiplatelet agent for use in patients presenting with ST-segment elevation myocardial infarction (STEMI) undergoing primary percutaneous coronary intervention (PPCI) because of its rapid onset of antiplatelet action. However, trials have suggested an increased bleeding risk with Prasugrel compared to Clopidogrel because of its increased relative potency leading to a recommendation that it should not be used in patients with high bleeding risk (HBR) - $>75 \mathrm{yrs}$ of age, $<60 \mathrm{~kg}$ in weight or a prior history of cerebrovascular accident (CVA).

Purpose There are some theoretical advantages in using Prasugrel for loading in all patients undergoing PPCI and switching to Clopidogrel (or low dose Prasugrel) for maintenance. Our STEMI protocol suggests Prasugrel loading for all comers undergoing PPCI on this basis (irrespective of bleeding risk). We examined the bleeding incidence and mortality in STEMI patients who underwent PPCI and received Prasugrel loading despite having HBR characteristics and compared it to those who received Clopidogrel (because of HBR) and those who did not have HBR characteristics (who received Prasugrel loading).

Abstract 59 Table 1 Table with incidence of bleeding on the 3 groups of patients based on BARC score

\begin{tabular}{|c|c|c|c|c|c|c|c|c|c|c|c|}
\hline & & Group & & & Group 2 & & & Group 3 & & & \\
\hline & BARC & $\begin{array}{c}\text { Score } \\
1\end{array}$ & $\begin{array}{c}\text { Score } \\
2\end{array}$ & $\begin{array}{c}\text { Score } \\
5\end{array}$ & Score 1 & score 2 & $\begin{array}{c}\text { Score } \\
3 a\end{array}$ & Score 1 & $\begin{array}{c}\text { Score } \\
2\end{array}$ & $\begin{array}{c}\text { Score } \\
3 a\end{array}$ & $\begin{array}{c}\text { Score } \\
3 b\end{array}$ \\
\hline Bleeding & $\begin{array}{l}\text { Pre- } \\
\text { Discharge }\end{array}$ & - & 1 & 1 & 1 & 1 & 2 & - & 1 & 4 & 1 \\
\hline & $\begin{array}{l}\text { Post- } \\
\text { discharge }\end{array}$ & 1 & - & - & 1 & 4 & - & 1 & 6 & - & - \\
\hline $\begin{array}{l}\text { No } \\
\text { bleeding }\end{array}$ & & 32 & & & 45 & & & & 193 & & \\
\hline Mortality & & $\begin{array}{c}7 \\
120 \%)\end{array}$ & & & $\begin{array}{c}4 \\
\mid 7.4 \%)\end{array}$ & & & $\begin{array}{c}11 \\
|5.33 \%|\end{array}$ & & & \\
\hline
\end{tabular}

